\title{
RESSONÂNCIA MAGNÉTICA DE ALTA RESOLUÇÃO ESPACIAL PARA ESTUDO ARTERIAL PERIFÉRICO COM AOUISIC̣̃̃O SEGMENTAR E CONTÍNUA: MOBITRAK*
}

\author{
Daniel L.S. Ribeiro' ${ }^{1}$, Mario M. Galvão Fo22, Giuseppe D’lppolito², Angela M.B. Wolosker², \\ Maria Lucia Borri ${ }^{2}$, Luiz de Abreu $\mathrm{Jr}^{3}{ }^{3}$, Luiz Guilherme Hartmann ${ }^{3}$, Adriano Fleury ${ }^{3}$
}

Resumo 0 objetivo é apresentar uma técnica recente para a avaliação de obstruções arteriais dos membros inferiores por ressonância magnética em um único momento e com a utilização de dose dupla do meio de contraste paramagnético administrado de forma lenta através de bomba injetora. 0 método baseia-se em um "software" e "hardware" denominado Mobitrak, disponível nos aparelhos de alto campo de ressonância da Philips, que permite a avaliação de uma grande extensão vascular a partir de aquisições de alta resolução, segmentares e contínuas. A seqüência utilizada é um gradiente eco (FFE) que permite a programação de três segmentos simultaneamente, com pequena sobreposição nas intersecções desses segmentos. Essa seqüência dinâmica é obtida em duas fases, uma previamente ao contraste e outra durante a injeção lenta deste, com subtração do sinal dos tecidos adjacentes e reconstruções em 3D. 0 método apresenta vários benefícios, como: melhor visualização dos segmentos tibiofibulares, estudo de toda a aorta e o membro inferior em uma única visita do paciente, com baixo volume de meio de contraste.

Unitermos: Angiorressonância magnética; Arterial; Técnica.

Abstract High-resolution magnetic resonance for peripheral arterial study with segmentar and continuous acquisition: Mobitrak.

Our purpose is to present a new technique to evaluate arterial obstructions in the lower limbs by magnetic resonance using a single paramagnetic contrast agent double dose slowly administered using an infusion pump. The method is based on a software and hardware called Mobitrak available on the Philips high field magnetic resonance machines, which allows vascular evaluation of wide areas with continuous and segmentar high resolution acquisitions. An echo gradient sequence (FFE) was used, which allowed three simultaneous planning and had little overlapping on the intersections. This dynamic sequence is achieved in two phases, prior and during contrast injection, and allows signal subtraction of adjacent tissues and 3D reconstructions. The method has various advantages such as better visualization of the tibiofibular segments, study of the whole lower limb in a single patient visit, and reduction of the volume of contrast injected.

Key words: Magnetic resonance angiography; Arterial; Technique.

\section{INTRODUÇÃO}

O desenvolvimento da angiografia por ressonância magnética (angio-RM) teve um avanço considerável nos últimos cinco a seis anos, e por isso tem-se tornado o método de escolha para a avaliação de pacientes com doenças arteriais ${ }^{(\mathbf{1})}$. Inicial-

\footnotetext{
* Trabalho realizado no Serviço de US/TC/RM do Complexo Hospitalar São Luiz, São Paulo, SP.

1. Médico Estagiário do Serviço de TC e RM do Complexo Hospitalar São Luiz.

2. Radiologistas do Serviço de TC e RM do Complexo Hospitalar São Luiz e do Departamento de Diagnóstico por Imagem da Universidade Federal de São Paulo/Escola Paulista de Medicina (Unifesp/EPM).

3. Radiologistas do Serviço de TC e RM do Complexo Hospitalar São Luiz.

Endereço para correspondência: Dr. Mario M. Galvão Fo. Hos pital São Luiz - Ressonância Magnética. Rua Engenheiro Oscar Americano, 840, Morumbi. São Paulo, SP, 05673-050. E-mail: mgalvaof@uol.com.br.

Recebido para publicação em 22/10/2003. Aceito, após revisão, em 22/12/2003.
}

mente, seqüências fluxo dependentes eram utilizadas para a realização dos estudos por angio-RM e foram gradativamente substituídas por sequiências rápidas e ultra-rápidas com utilização do meio de contraste paramagnético, demonstrando superioridade técnica na avaliação de obstruções $\operatorname{arteriais~}^{(1-3)}$.

A avaliação dos vasos abdominais era limitada a um único segmento com uma fase arterial. Recentemente, o avanço nas seqüências de pulso associado ao desenvolvimento da capacidade dos "hardwares" determinaram a redução drástica nos tempos das sequiências, permitindo a aquisição de múltiplos segmentos em até uma única apnéia ${ }^{(\mathbf{1})}$. Dessa forma, a avaliação de obstruções aórticas e arteriais dos membros inferiores por RM em um único momento e com a utilização de dose dupla do meio de contraste paramagnético administrado de forma lenta através de bomba injetora tem sido utilizada cada vez mais, uma vez que apresenta acurácia bastante elevada. O nosso objetivo é apresentar esta técnica, com exemplificação de alguns casos do nosso dia-a-dia.

$\mathrm{O}$ estudo por "moving-bed infusiontracking MR angiograms" (Mobitrak) consiste numa avaliação pré-contraste e outra durante a infusão deste. Realiza-se, após as aquisições, uma subtração para eliminar os tecidos de partes moles adjacentes, destacando-se os vasos contrastados.

Os fundamentos básicos do método consistem na infusão lenta do meio de contraste, contrariamente ao realizado até pouco tempo, quando grandes volumes de contraste eram injetados em bolo e no posicionamento adequado do paciente. 
Durante a infusão, uma sequiência gradiente eco é adquirida mostrando a árvore vascular da aorta ao arco podálico. Esta ampla cobertura é obtida através de três segmentos numa única aquisição e com dose dupla de injeção, sendo um método rápido e que permite uma excelente avaliação da anatomia vascular, desde a aorta até os arcos podálicos em uma única visita do paciente.

\section{TÉCNICA}

O procedimento consiste em quatro etapas: posicionamento adequado do paciente na mesa de exame, determinação da anatomia vascular com o uso prévio da sequiência TOF, determinação do melhor momento de aquisição e a aquisição durante a injeção de gadolínio com movimentação sincrônica da mesa de exame.

Inicia-se pelo posicionamento, que é muito importante e exige a máxima atenção, pois as seqüências são muito sensíveis a movimentos. O paciente é colocado em decúbito dorsal, com os joelhos levemente fletidos num mesmo plano dos tornozelos. Os pés são dispostos com o toque dos calcanhares e afastamento das pontas dos pés, fazendo um ângulo de aproximadamente 60 graus de abertura. Existem ainda duas faixas que fixam os joelhos e tornozelos, determinando leve compressão das veias do sistema profundo, impedindo o fluxo venoso profundo que poderia causar uma leitura equivocada por se interpretar erroneamente uma veia profunda como sendo uma artéria. Para este posicionamento existe um suporte específico e ajustável para diversas alturas dos pacientes (Figura 1).

Reconstruções sagitais "maximum intensity projection" (MIP) são obtidas através de seqüências TOF. Estas imagens MIP são utilizadas para obterem-se informações sobre o curso dos vasos arteriais em direção ântero-posterior, consistindo em três seqüências não sincronizadas cobrindo a árvore vascular dos pés à aorta. Para obter-se um exato alinhamento destas imagens, algumas precauções devem ser tomadas, como mover a mesa em posições predeterminadas, manter os membros inferiores fixos para evitar movimentação, além de boa orientação ao paciente. $\mathrm{O}$ pri-

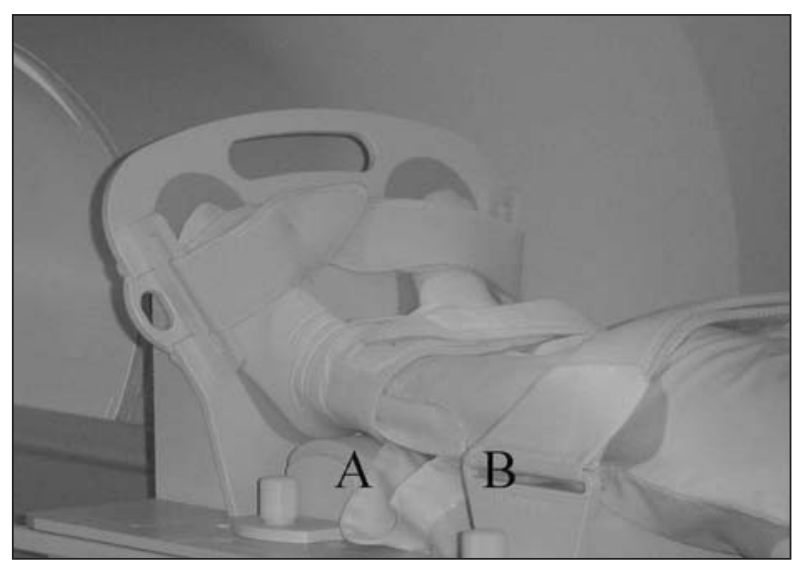

Figura 1. Suporte para o po sicionamento dos membros inferiores com compressão nos calcanhares $(A)$ e regiões poplíteas (B) para evitar refluxos venosos pelo sistema profundo. meiro volume é planejado para avaliar as artérias da região abdominal inferior, e movendo-se a mesa, captam-se as artérias das outras regiões (coxa e perna) dentro do volume de imagem, conseguindo-se tradução de toda a árvore vascular periférica sem reposicionar o volume adquirido (no sentido ântero-posterior) ${ }^{(3)}$.

A determinação do melhor momento de aquisição é feita através de teste-dose ou de "bolus track". O primeiro, e o de nossa preferência, consiste na aquisição rápida de vários "scans" em um mesmo ponto localizado ao nível da porção distal da aorta abdominal. Durante a infusão de 2 $\mathrm{ml}$ de contraste seguida de $10 \mathrm{ml}$ de soro fisiológico procura-se identificar o tempo em que ocorre a melhor visualização do vaso escolhido (Figura 2). Portanto, o tempo de "delay" a ser utilizado na aquisição do angiograma é determinado pelo primeiro "scan" em que ocorre o maior realce da $\operatorname{aorta}^{(3)}$. A outra forma, ou seja, o "bolus track", baseia-se na observação em tempo real do momento de maior realce da aorta a partir do qual se inicia a aquisição.

A dose de contraste preconizada para o exame é de $0,8 \mathrm{ml} / \mathrm{kg}$ (dose dupla), até o máximo de $40 \mathrm{ml}$. Utiliza-se infusão lenta e progressiva através de bomba injetora, iniciando-se com velocidade de $0,6 \mathrm{ml} / \mathrm{s}$ para a aorta seguida por $0,3 \mathrm{ml} / \mathrm{s}$ para os segmentos distais. A importância desta infusão reside no fato de se necessitar de uma manutenção contínua e uniforme no padrão de realce durante toda a aquisição. Deve-se estar atento ao fato de que a injeção ideal deve ocorrer por pelo menos dois terços do tempo total de aquisição ${ }^{(3)}$.

$\mathrm{Na}$ seqüência utilizada, o TR e o TE devem ser encurtados ao máximo e um FOV ("field of view") retangular deve ser reduzido ao mínimo. Este artifício pode, muitas vezes, determinar o aparecimento de artefatos que rebatem as laterais do corpo para o centro da imagem. Para evitá-lo, utilizam-se duas bandas de saturação com $2,0 \mathrm{~cm}$ de espessura em cada la-

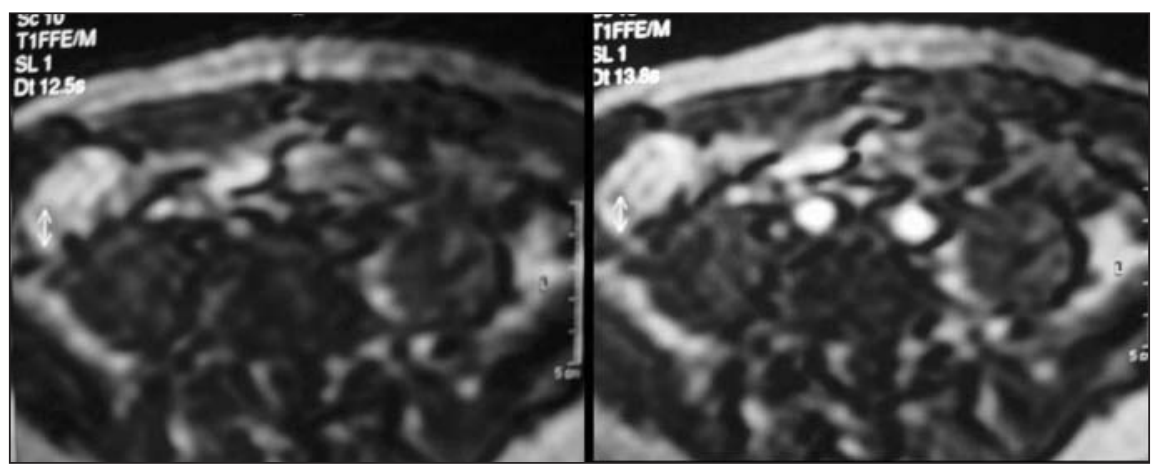

Figura 2. Teste-dose. Na imagem de RM à esquerda observa-se plano na bifurcação da aorta sem contraste após 12,5 segundos do início da injeção. À direita, com 13,8 segundos, temos realce arterial ideal, indicando o tempo de início da aquisição. 
teral do corpo ${ }^{(3)}$. Com esses parâmetros consegue-se tempo de aquisição de cerca de 40 segundos por segmento (dois minutos e 40 segundos em todo o estudo dinâmico, incluindo-se os tempos de preparação). Isto resulta em ganho de tempo (20 minutos) para adquirir as seqüências TOF e suas imagens MIP sagitais. Utilizamos, em nossos exames, o seguinte protocolo: $\mathrm{FOV}=430, \mathrm{RFOV}=75, \mathrm{TE}=1,6, \mathrm{TR}=$ 5,7 , "flip" $=35$, matriz $=512 \times 512$ (aquisição e reconstrução), "scan dyn" = 1:20. A seqüência de angio-RM pós-gadolínio e com movimentação da mesa de exame baseia-se na técnica gradiente-eco. As imagens são adquiridas continuamente e com pequena sobreposição nas intersecções dos segmentos.

O método denominado iô-iô, tradução do movimento sequiencial da mesa para dentro e para fora, define da forma mais fiel possível como é realizada a aquisição das imagens. Após o posicionamento do paciente, marca-se o tornozelo como ponto zero, que é deslocado para o interior do magneto. Inicia-se a aquisição de um localizador-"surview" para o planejamento do estudo dinâmico, em um movimento seqüencial no sentido crânio-caudal. No momento seguinte, imagens pré-contraste que serão utilizadas como máscara são adquiridas em um movimento similar ao descrito anteriormente, agora em sentido oposto (caudo-cranial). A aquisição das máscaras é sugerida pela literatura em todos os segmentos, pois quando são subtraídas das imagens pós-contraste, obtémse qualidade similar às angiografias digitais ${ }^{(\mathbf{1 - 8})}$. Após a aquisição das imagens précontraste, a preparação do estudo contrastado é iniciada, sendo o procedimento idêntico, só que novamente em sentido crânio-caudal, iniciando-se a aquisição da aorta seguida da região crural e, por fim, das pernas.

Finalizando, realiza-se a subtração dos estudos dinâmicos de cada segmento, individualmente. As imagens pós-subtração são enviadas para uma estação de trabalho (Workstation Easy Vision, Philips) onde são realizadas as reconstruções MIP em três dimensões, no plano coronal. Um total de 12 imagens por segmento arterial é documentado ao longo do seu eixo crâniocaudal em angulações diferentes, evitando-

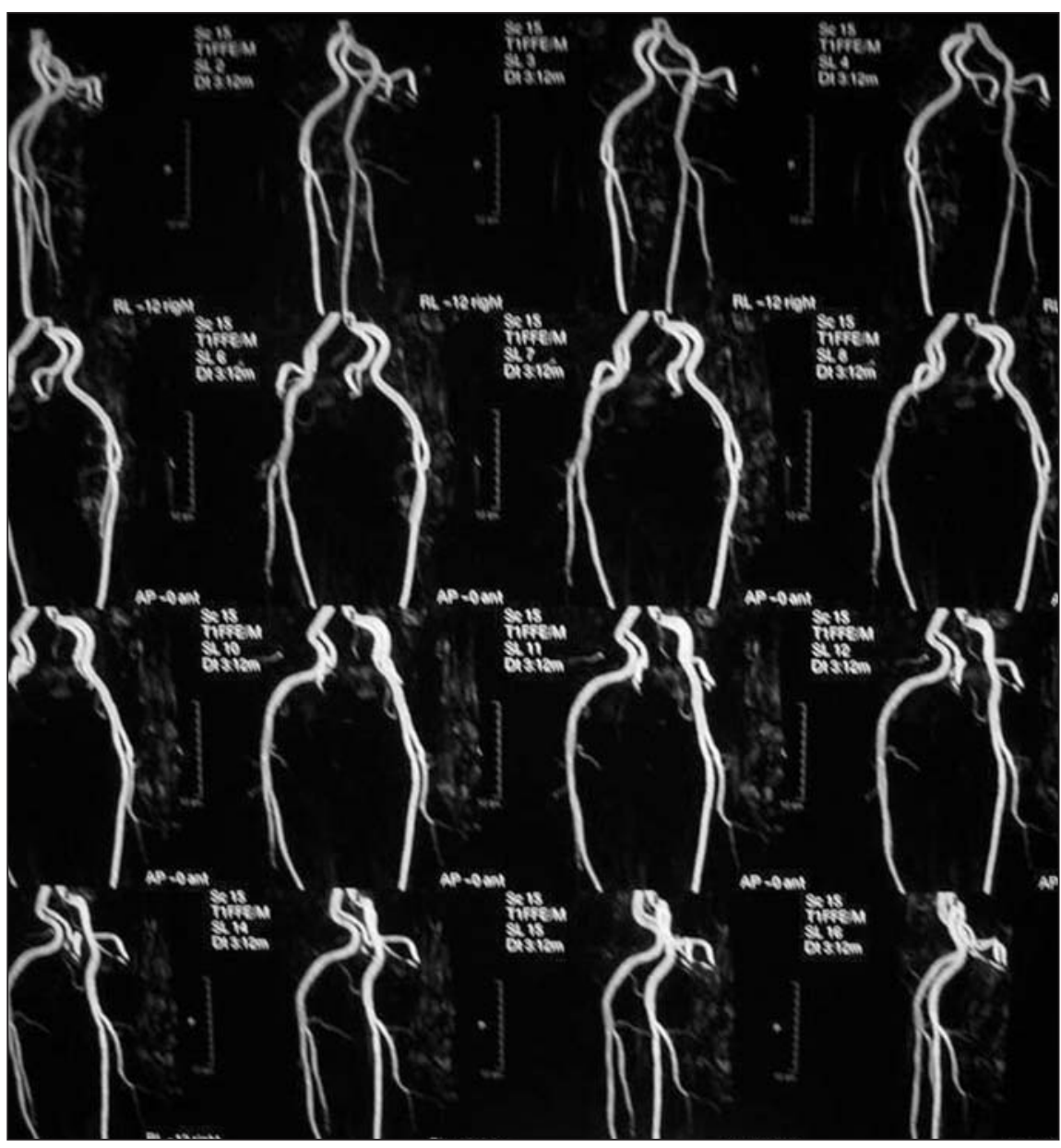

Figura 3. Reconstruções MIP-3D em diferentes angulações, nos planos coronais, permitindo uma leitura sem sobreposições das artérias ilíacas.

se sobreposições (Figura 3). A última etapa de manipulação das imagens consiste em documentar em um único filme e de forma contínua os três segmentos arteriais (Figuras 4, 5, e 6).

\section{DISCUSSÃO}

O desenvolvimento da angio-RM, recentemente, tem melhorado consideravelmente a avaliação dos pacientes com doença vascular, e a angio-RM tem-se tornado o exame de escolha na maioria dos $\operatorname{casos}^{(\mathbf{1})}$. As aquisições pós-injeção do meio de contraste (gadolínio) mostram vantagens em relação à seqüência TOF, como maior resolução espacial, rápida aquisição e maior relação sinal/ruído ${ }^{(4)}$. Na técnica apresentada, combinam-se as vantagens das aquisições pós-gadolínio 3D com a movimentação da mesa, como utilizado na angiografia convencional. Em 1998, Ho et al. compararam angiografia digital com angioRM pós-gadolínio com movimentação da mesa, tendo mostrado excelente correlação na identificação de estenoses significativas ou não, com baixa variação interobservador e superioridade na definição de oclusão de pequenos ramos vasculares ${ }^{(3)}$. Levando-se em consideração estes achados e outros citados na literatura, é substancialmente mais segura e menos dispendiosa a realização da angio-RM em vez da angiografia digital com a utilização do meio de contraste iodado, além da possibilidade em se manipular as imagens da RM com reconstruções 3D, endonavegação.

Meaney et al., em estudo semelhante, obtiveram sensibilidade de $81 \%$ e especificidade de $95 \%$ na demonstração de estenoses arteriais significativas (>50\%) ou não $(<50 \%)$, mostrando ainda sensibilidade e especificidade de $94 \%$ e $97 \%$, respectivamente, na definição de oclusões ${ }^{(4)}$. 


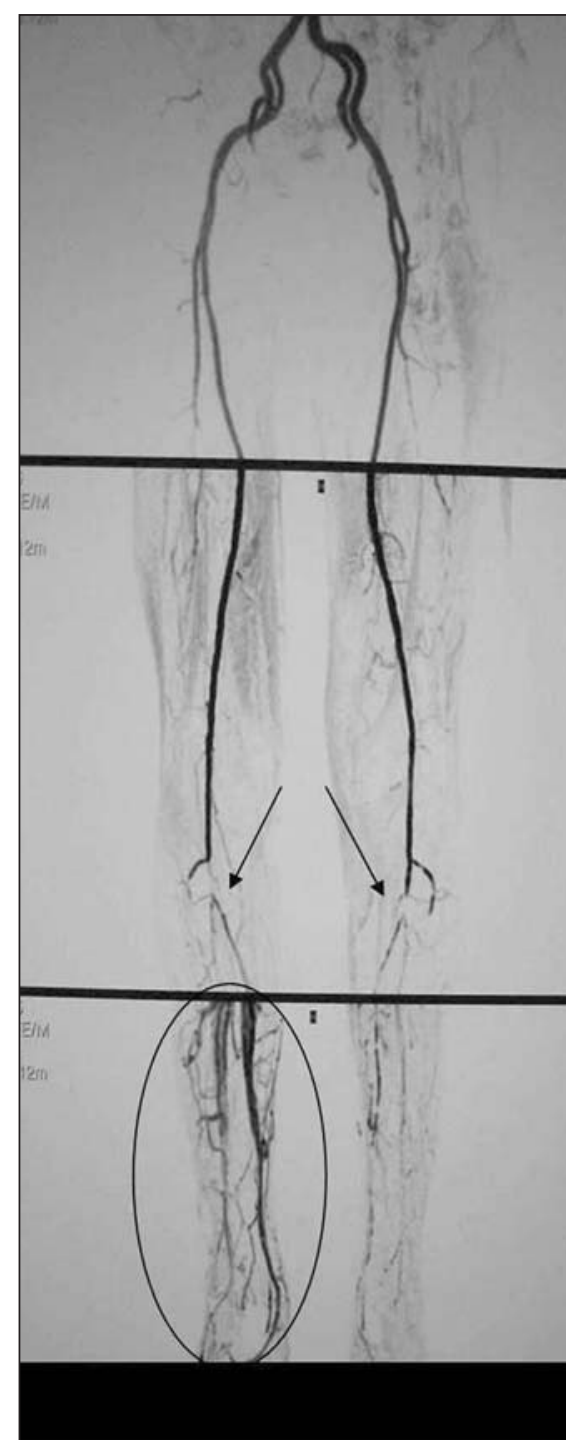

Figura 4. Documentação final de angio-RM com Mobitrak, em que se pode observar obstruções arteriais ao nível das poplíteas (setas), sendo maior à direita. Na porção distal do membro inferior direito existe importante circulação colateral compensatória (0).

Atualmente já é possível o estudo arterial de todo o corpo, desde as carótidas até os arcos podálicos, com sensibilidade de $95,3 \%$ e especificidade de $95,2 \%$ na detecção de estenoses significativas (> 50\%) na doença arterial periférica ${ }^{(6)}$.

Dessa maneira, esta técnica de angioRM com movimentação sincronizada da mesa de exame e utilização de dupla dose de contraste permite o estudo vascular desde a aorta até o tornozelo, de maneira não-invasiva, com alta acurácia, indicando uma ótima relação custo/benefício para os pacientes.
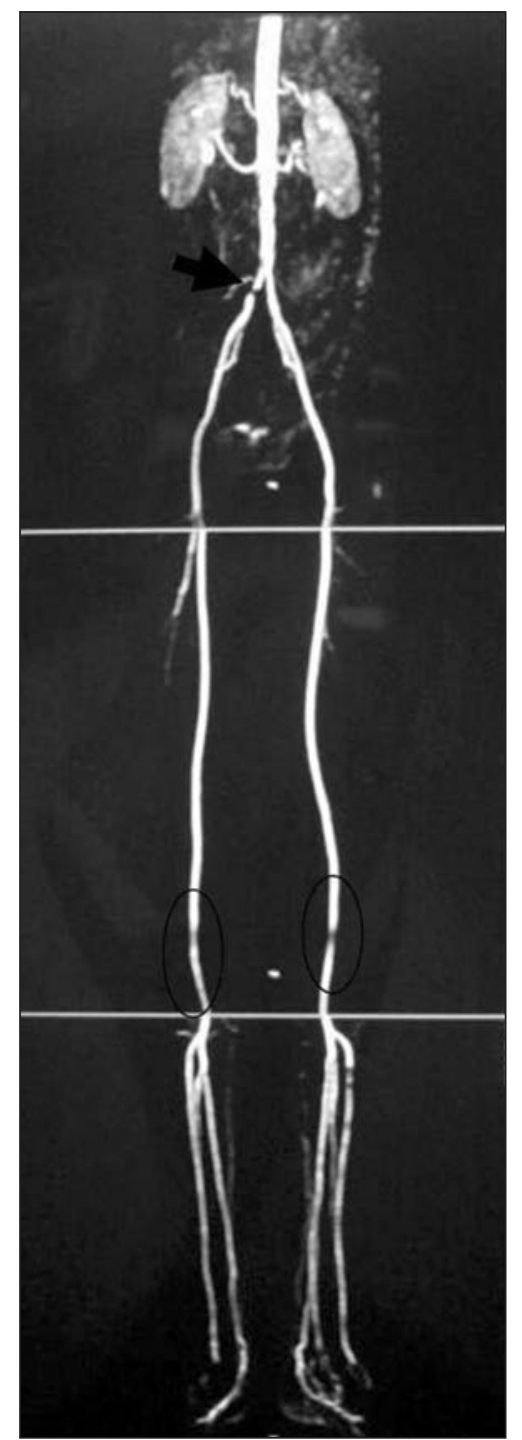

Figura 5. Reconstruções de exame de um paciente com dor crônica no membro inferior direito, onde a angio-RM mostrou estenose significativa ao nível da artéria ilíaca comum (seta). Observar os artefatos determinados por compressão do suporte, para evitar fluxo venoso, ao nível das artérias poplíteas (0).

\section{REFERÊNCIAS}

1. Shetty AN, Bis KG, Duerinckx AJ, Narra VR. Lower extremity MR angiography: universal retrofitting of high-field-strength systems with stepping kinematic imaging platforms initial experience. Radiology 2002;222:284-91.

2. Foo TK, Ho VB, Hood MS, Marcos HB, Hess SL, Choyke PL. High spatial resolution multistation MR imaging of lower-extremity peripheral vasculature with segmented volume acquisition: feasibility study. Radiology 2001;219:835-41.

3. Ho KY, Leiner T, de Haan MW, et al. Peripheral vascular tree stenoses: evaluation with moving-bed infusion-tracking MR angiography. Radiology 1998; 206:683-92.

4. Meaney JF, Ridgway JP, Chakraverty S, et al. Stepping-table gadolinium-enhanced digital subtraction MR angiography of the aorta and lower extremity

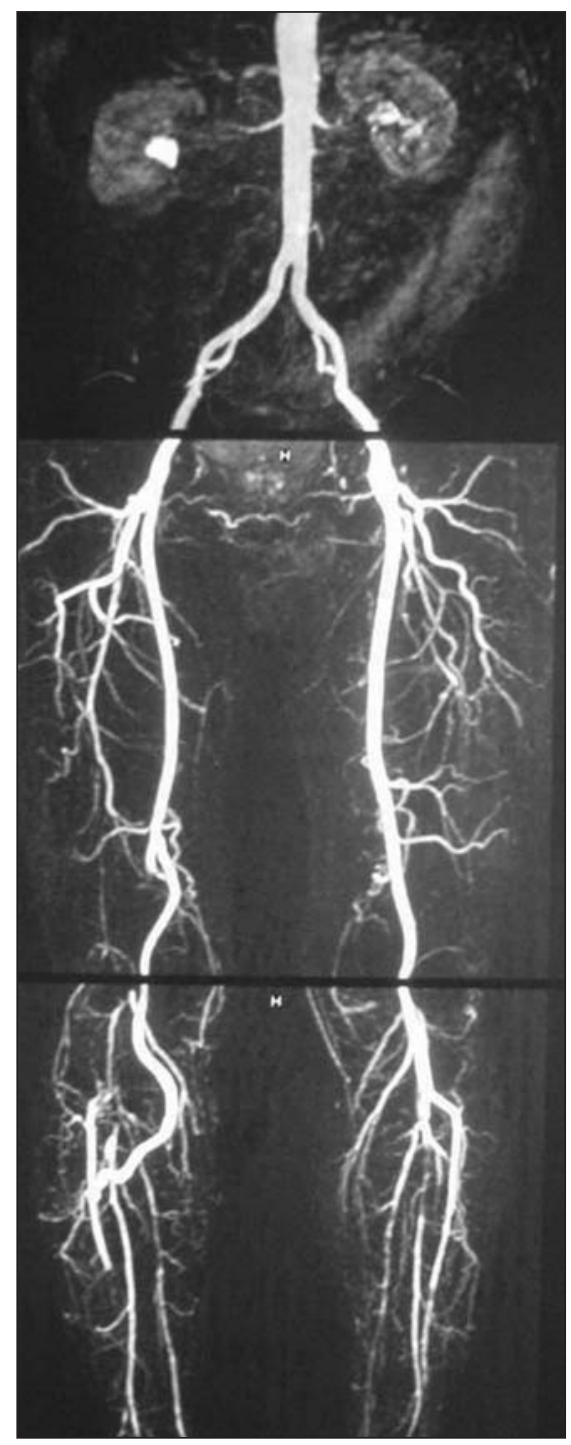

Figura 6. Paciente com obstrução arterial crônica na artéria femoral superficial do membro inferior direito, com intensa circulação colateral

arteries: preliminary experience. Radiology 1999 211:59-67.

5. Earls J, DeSena S, Bluemke DA. Gadolinium-enhanced three-dimensional MR angiography of the entire aorta and iliac arteries with dynamic manual table translation. Radiology 1998;209:844-9.

6. Goyen M, Quick HH, Debatin JF, et al. Whole-body three-dimensional MR angiography with a rolling table platform: initial clinical experience. Radiology 2002;224:270-7.

7. Earls JP, Patel NN, Smith PA, et al. Gadoliniumenhanced three-dimensional MR angiography of the aorta and peripheral arteries: evaluation of a multistation examination using two gadopentetate dimeglumine infusions. AJR 1998;171:599-604

8. Wang Y, Lee HM, Khilnani NM, et al. Bolus-chase MR digital subtraction angiography in the lower extremity. Radiology 1998;207:263-9. 